\title{
Differentiation of Cardiomyocytes and Identification of Cardiac Conduction System Connexins Derived from Bone Marrow Mesenchymal Stem Cells of Macaca nemestrina
}

\author{
Agus Harsoyo $^{1 *}$, Irma Herawati Suparto ${ }^{2}, Y_{0 g a}$ Yuniadi $^{3}$, Arief Boediono ${ }^{4}$, Dondin Sajuthi ${ }^{5}$ \\ ${ }^{1}$ Primate Research Center (PSSP), Bogor Agricultural University, Bogor, Indonesia \\ ${ }^{2}$ Department of Chemistry, Faculty of Math and Science, Bogor Agricultural University, Bogor, Indonesia \\ ${ }^{3}$ Department of Cardiology and Vascular Medicine, Faculty of Medicine, Universitas Indonesia, Jakarta, Indonesia \\ ${ }^{4}$ Department of Veterinary Clinic Reproduction and Pathology, Faculty of Veterinary Medicine, Bogor Agricultural University, Bogor, \\ Indonesia \\ ${ }^{5}$ Department of Anatomy, Physiology, and Pharmacology, Faculty of Veterinary Medicine, Bogor Agricultural University, Bogor, \\ Indonesia
}

ARTICLE INFO

\section{Article history:}

Received December 27, 2018

Received in revised form August 30, 2020

Accepted September 15, 2020

\section{KEYWORDS:}

bone marrow mesenchymal stem cells, cardiac conduction system connexin, isolation and differentiation, pigtail macaques

\begin{abstract}
Bone marrow mesenchymal stem cells have been widely used, because plasticity, specific surface markers, self-renewal to transform into various lineages including cardiomyocytes. Information about the connexin $(\mathbf{C x})$ cardiac conduction systems of the pigtail macaque (Macaca nemestrina) is limited. This study aimed to evaluate cardiomyocyte differentiation from bone marrow mesenchymal stem cells of pigtail macaques and to clarify the $\mathbf{C x}$ cardiac conduction system. Bone marrow aspirates were obtained from the proximal humerus of four adult male pigtail macaques, collected into heparinized tubes, then centrifuged to obtain mononuclear cells that were isolated and cultured in an incubator. After these cells reached $70-80 \%$ monolayer confluency as homogeneous fibroblast-like cells, they were subcultured. On the second subculture passage, the cells were pelleted to extract the mRNA, which was analysed by reverse transcription-polymerase chain reaction, and then cultured for a third passage. Cells were positive for $\mathrm{CD} 73$ and CD105 and the reference gene glyceraldehyde-3-phosphate dehydrogenase, and negative for CD34 and CD45. Osteogenic, chondrogenic, adipogenic, and cardiomyocyte differentiation was confirmed based on specific staining. The pigtail macaque bone marrow mesenchymal stem cells can be isolated and subcultured. The transcription of genes and translation of proteins of the connexin cardiac conduction systems was successfully identified.
\end{abstract}

\section{Introduction}

About 100,000-200,000 primates are used annually for research in the United States, Europe and Japan. The Macaca genus is often selected for biomedical research because of its similarities to humans in terms of physiological, anatomical and genetic composition, which is around 93.5\% identical with humans. The species Macaca nemestrina from Indonesia used in this study is spread across the peninsula of Indochina, Sumatra, and Borneo. There are no data on the use of $M$. nemestrina from Indonesia for the study of cardiomyocyte differentiation and

\footnotetext{
* Corresponding Author

E-mail Address: ags_harsoyo@yahoo.com
}

the connexin $(\mathrm{Cx})$ electrical conduction system of the heart. This is important because samples from different geographic origins may yield different results (Carlsson et al. 2004).

The concept of using bone marrow cells for research was initiated by Maximov, who identified haematopoietic cells and haematopoietic stem cells, while Friedenstein discovered that bone marrow also contained non-haematopoietic stem cells of bone marrow origin (Friedenstein 1989). Mesenchymal stem cells are widely used because they can easily be isolated and developed into various differentiated cell types. The minimum criteria for defining mesenchymal stem cells are: cells that are capable of adhering to the substrate upon which they grow, that express cluster differentiation (CD) 105 and 
CD73, and do not express CD34 and CD45, and can differentiate in vitro into osteocytes, chondrocytes, adipocytes, and cardiomyocytes (Dominici et al. 2006). Differentiation into cardiomyocytes has been studied in the M. mullata model (Zhang et al. 2017) but there are no available data on the translation of cardiac electrical system protein Cx. Existing data on Cx-expressing cardiac electrical cells are limited to rabbits (Boyett et al. 2006), guinea pigs, pigs, cows (van Kempen et al. 1995) and humans (Antonitsis et al. 2007).

Cardiomyocytes make up around 75\% of the myocardium and act as an electrical conduction system for contraction, automatic rhythmic motion, intercellular conduction and electromechanical coupling. Morphological changes in cardiomyocytes affect the deepest connective structures that cause cardiac electrical rhythm abnormalities. A group of myocytes is wrapped by intercalating discs connected by desmosomes, adherent junctions and gap junctions. Structurally, the endothelial layer of a gap junction is made up of Cx40, Cx43, Cx45, and $\mathrm{Cx} 37$. Studies show that the expression of $\mathrm{Cx} 40$ and $\mathrm{Cx} 43$ in the His bundles of pigs is the same as that in humans; whereas those in mice, guinea pigs and cows differ from those of humans. In human atrioventricular nodes, the mRNA expressed is mostly that of $\mathrm{Cx} 40$, with a small amount of $\mathrm{Cx} 43$ (Temple et al. 2013).

There are limited data available about differentiated cardiomyocytes derived from mesenchymal stem cells of bone marrow origin, and antibody-mediated identification of $\mathrm{Cx} 40$ and Cx43 expression on cardiac electrical cells of $M$. nemestrina from Indonesia has not been performed. Therefore, the aim of this study was to isolate and develop mesenchymal stem cells obtained from $M$. nemestrina bone marrow aspirates, differentiate them into cardiomyocytes and identify cardiac electrical cells expressing $\mathrm{Cx} 40$ and $\mathrm{Cx} 43$ in vitro. The results of this study may be useful for the therapy of cardiac electrical abnormalities in the atrioventricular node.

\section{Materials and Methods}

\subsection{Experimental Animals}

This study used samples of bone marrow aspirates from four adult male $M$. nemestrina aged 5-7 years old with a body weight of $6-8 \mathrm{~kg}$. The procedures in this study were approved by the Research Animal
Welfare and Usage Monitoring for Breeding and Education Commission from the Institut Pertanian Bogor (Agricultural Institute of Bogor) Primate Study Center, approval number IPB PRC-18-B001.

\subsection{Research Materials}

Cell culture growth media materials included phosphate-buffered saline (PBS) washing medium (Gibco, USA) and trypsin (Sigma, USA). Materials for evaluating the differentiation of mesenchymal stem cells included RNeasy kits (Qiagen, USA), SuperScript III Reverse Transcriptase kit (Invitrogen, USA), agarose, tris/acetic acid/ethylenediaminetetraacetic acid (TAE) buffer solution, and polymerase chain reaction (PCR) reagents. Primers used were for CD73 and CD105, markers of mesenchymal stem cells, the haematopoietic stem cell markers CD34, CD45 and the reference gene glyceraldehyde-3-phosphate dehydrogenase (GAPDH). The staining reagents used were alizarin red, Alcian blue, oil red 0 and 5-aza-2'deoxycytidine (all obtained from ScienCell, USA).

\subsection{Research Procedure}

\subsubsection{Bone Marrow Collection}

The bone marrow was collected from M.nemestrina caput humerus with an $18 \mathrm{G}$ needle. The aspirate was collected into a heparin-containing tube, then layered over Ficoll and subsequently centrifuged at $800 \mathrm{~g}$ for 30 minutes. The buffy coat layer was harvested into PBS washing medium, centrifuged again at $500 \mathrm{~g}$ for 15 minutes and then washed twice more. Mononuclear cells were stained with Trypan blue and counted using a haemocytometer. Other cells were cultured on six-welled culture plates with growth medium. Incubation was carried out at $37^{\circ} \mathrm{C}$, $5 \% \mathrm{CO}_{2}$ and cells were passaged when they reached $70-80 \%$ confluence.

\subsection{2. mRNA Extraction and PCR Amplification of Stem Cell Markers}

The extraction of mRNA and reverse transcriptionPCR (RT-PCR) were performed according to the manufacturer's recommended protocols. PCR reagents consisted of PCR 10x buffer solution, $25 \mathrm{mM} \mathrm{MgCl}$, $10 \mathrm{mM}$ dNTP, Taq gold polymerase, nuclease-free water, primers (forward and reverse) and 2-5 ng complementary deoxyribonucleic acid (cDNA) samples. The expression of mesenchymal stem cell markers and haematopoietic stem cells was assessed after 40 cycles of PCR. PCR products were electrophoresed on $2 \%$ agarose containing ethidium bromide in TAE buffer 
solution at 100 volts. Visualization of electrophoresis results was performed using the Gel Doc documentation tool.

\subsubsection{Procedures for Osteogenic, Chondrogenic and Adipogenic Differentiation}

Cells were cultured at 3,000 cells $/ \mathrm{cm}^{2}$ in 12-welled cell culture plates and incubated for 18-20 hours. Subsequently, cells were grown on osteogenic differentiation medium consisting of Dulbecco's Modified Eagle Medium (DMEM), 10\% foetal bovine serum (FBS), $50 \mu \mathrm{g} / \mathrm{ml}$ ascorbic acid, $10^{-7} \mathrm{M}$ dexamethasone and $10 \mathrm{mM}$ glycerol phosphate. Cells were cultured in this medium for 21 days at $37^{\circ} \mathrm{C}$, with a $\mathrm{CO}_{2}$ concentration of $5 \%$, and the medium was replaced every 3 days. The medium was then discarded and cells were washed with PBS and fixed with $10 \%$ paraformaldehyde for 30 minutes. Plates were washed with PBS and stained for 1 hour with alizarin red to detect osteogenic differentiation, washed again with PBS and observed under an inverted microscope. Alcian blue staining was used to detect chondrogenic differentiation and oil red $\mathrm{O}$ staining was used to detect adipogenic differentiation.

\subsubsection{Procedures for Cardiomyocyte Differentiation}

Cells were cultured at a concentration of 500 cells/ $\mathrm{cm}^{2}$ in each of the 8-well slide chambers in mesenchymal stem cell growth medium at a temperature of $37^{\circ} \mathrm{C}$ and $5 \% \mathrm{CO}_{2}$ for $18-20$ hours. Cell cultures were then supplemented with cardiomyocyte differentiation medium containing 5-aza-2'-deoxycytidine(5-aza) and incubated for 16-20 hours. Incubation was continued in heart muscle cell differentiation medium without 5-aza, and the medium was replaced every 3-4 days. On day 21, staining of the cells was conducted using immunocytochemistry for $\mathrm{Cx} 40$ and $\mathrm{Cx} 43$.

\subsection{Analysis of Results}

Descriptive analysis included the morphology of circular mesenchymal stem cells, slightly elongated cells resembling fibroblasts, positive or negative staining for mesenchymal stem cell surface antigens and the presence or absence of expression of mRNA for mesenchymal stem cell markers. We examined markers for morphology and differentiation, i.e. calcium deposits for osteogenic differentiation, chondrocyte nodules for chondrogenic differentiation and lipid deposits for adipogenic differentiation. Differentiation into cardiomyocytes was analysed based on morphology and expression of proteins $\mathrm{Cx} 40$ and $\mathrm{Cx} 43$.

\section{Results}

\subsection{Bone Marrow Collection}

The number of mononuclear cells in the bone marrow aspirate was not proportional to the total volume of aspirate (Mn\#2 and Mn\#3) (Table 1).

Mononuclear cells were obtained through centrifugation; the buffy coat was collected while the red blood cells, granulocytes, immature myeloid cells, and platelets were discarded. It is estimated that the bone marrow mesenchymal stem cells constituted about $0.001-0.01 \%$ of the total mononuclear cells. On day 7 of culture, Mn\#1, Mn\#2, and Mn\#4 mononuclear cell cultures were $70-80 \%$ confluent, and the cell viability and total number of cells were counted. However, the Mn\#3 cells did not grow or develop (Table 2).

The formation of fibroblast spindle colonies, reflecting the development of mesenchymal stem cells, occurred after 24 hours in subcultures originating from

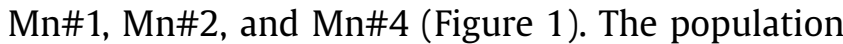
doubling time (PDT) of cells increased from the first to the third passage, and growth ceased after the third passage (Table 3 ).

Although the cells that developed had a high proliferative ability with viability $90.90-96.42 \%$, the number of cells decreased from $1,250,000$ to 120,000 and had a prolonged PDT of 3.62-9.60 days. The calculation of PDT was based on the time taken to double the cell numbers after isolation of the primary cell culture in vitro. The cell growth characteristics were expressed as cell lineage growth curves.

\subsection{PCR Amplification of Markers of Mesenchymal Stem Cells and Haematopoietic Stem Cells}

Extraction of mRNA from $M$. nemestrina bone marrow mesenchymal stem cells was successful. A total of $10 \mu \mathrm{lmRNA}$ was successfully converted into cDNA, which was then successfully amplified by PCR using primers for the stem cell markers (Figure 2 ). The PCR results showed that $M$. nemestrina bone marrow mesenchymal stem cells were positive for CD73 and CD105 markers. It can be seen that in cells from passage 2 , the size of the CD34 and CD45 bands was doubled, although mesenchymal stem cells do not express CD34 or CD45. 
Table 1. The number of mononuclear cells harvested from M. nemestrina bone marrow aspirates

\begin{tabular}{lcr}
\hline Animal id & $\begin{array}{c}\text { Volume of bone } \\
\text { marrow aspirate }(\mathrm{ml})\end{array}$ & $\begin{array}{l}\text { Number of } \\
\text { cells/well }\end{array}$ \\
\hline $\mathrm{Mn} \# 1$ & 8 & $8.83 \times 10^{6}$ \\
$\mathrm{Mn} 2$ & 5 & $10.25 \times 10^{6}$ \\
$\mathrm{Mn} \# 3$ & 5 & $6.00 \times 10^{6}$ \\
$\mathrm{Mn} \# 4$ & 9 & $10.38 \times 10^{6}$ \\
\hline
\end{tabular}

Mn: M. nemestrina

Table 2. Cell viability (\%) and number of mononuclear cells on day 7 of culture

\begin{tabular}{lcc}
\hline Animal id & Viability (\%) & $\begin{array}{l}\text { Number of } \\
\text { cells }\end{array}$ \\
\hline Mn\#1 & 98.00 & $5.40 \times 10^{6}$ \\
Mn\#2 & 93.34 & $7.80 \times 10^{6}$ \\
Mn\#3 & 96.62 & $4.80 \times 10^{6}$ \\
\hline
\end{tabular}

Mn: M. nemestrina

\subsection{Osteogenic, Chondrogenic andAdipogenic Differentiation}

Differentiation of $M$. nemestrina bone marrow mesenchymal stem cells into osteogenic, chondrogenic and adipogenic cells was successfully achieved. The staining for each differentiation lineage is shown in Figure 3.

Osteogenic differentiation produced cells with an elongated cuboid morphology. Differentiated osteocytes were stained with alizarin red, which indicated the presence of calcium-rich deposits. In contrast, chondrogenic differentiation produced cells that were shortened, enlarged and rounded at one end. Meanwhile, the cells in the control medium maintained the morphology of mesenchymal stem cells and grew accordingly. The differentiated
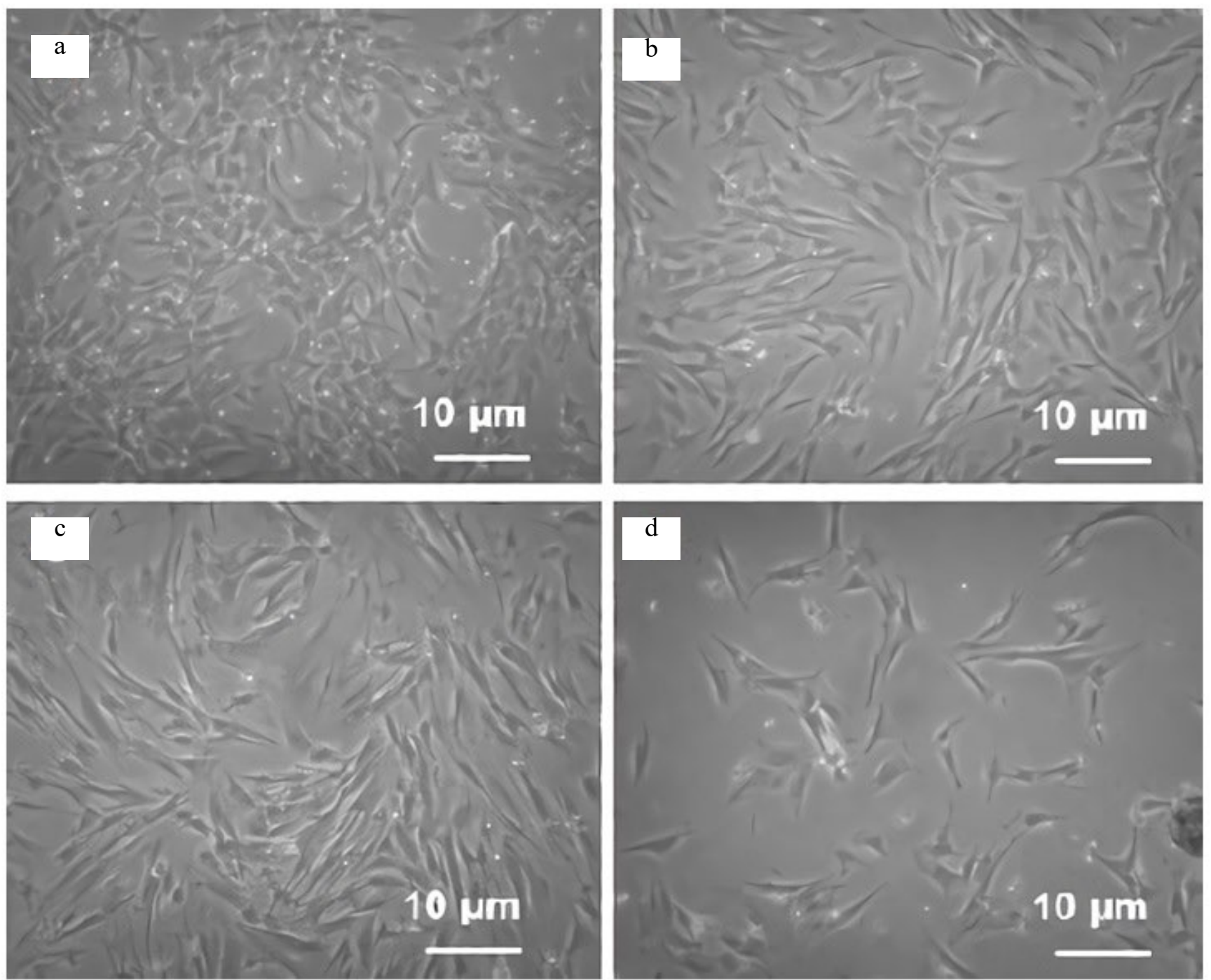

Figure 1. Mesenchymal stem cells of Macaca nemestrina bone marrow (a) P0, (b) P1, (c) P2, and (d) P3. (P refers to passage). Bar scale of $10 \mu \mathrm{m}$. 
Table 3. Cell viability (\%), number of cells and cellular population doubling time (PDT) for each passage (P)

\begin{tabular}{|c|c|c|c|c|c|c|c|c|c|}
\hline \multirow[t]{2}{*}{ Animal id } & \multicolumn{3}{|c|}{ Viability (\%) } & \multicolumn{3}{|c|}{ Number of cells } & \multicolumn{3}{|c|}{ PDT (days) } \\
\hline & P1 & P2 & P3 & P1 & $\mathrm{P} 2$ & P3 & P1 & P2 & P3 \\
\hline Mn\#1 & 93.83 & 94.59 & 90.90 & $1.90 \times 10^{6}$ & $1.2 \times 10^{5}$ & $2.0 \times 10^{5}$ & 3.62 & 5.73 & 6.14 \\
\hline $\mathrm{Mn} \# 2$ & 94.34 & 93.61 & 92.86 & $1.25 \times 10^{6}$ & $1.2 \times 10^{5}$ & $2.6 \times 10^{5}$ & 5.54 & 6.15 & 8.04 \\
\hline $\mathrm{Mn} \# 4$ & 96.42 & 94.11 & 91.67 & $2.00 \times 10^{6}$ & $9.6 \times 10^{5}$ & $3.3 \times 10^{5}$ & 7.40 & 9.60 & 9.48 \\
\hline
\end{tabular}

Mn: M. nemestrina

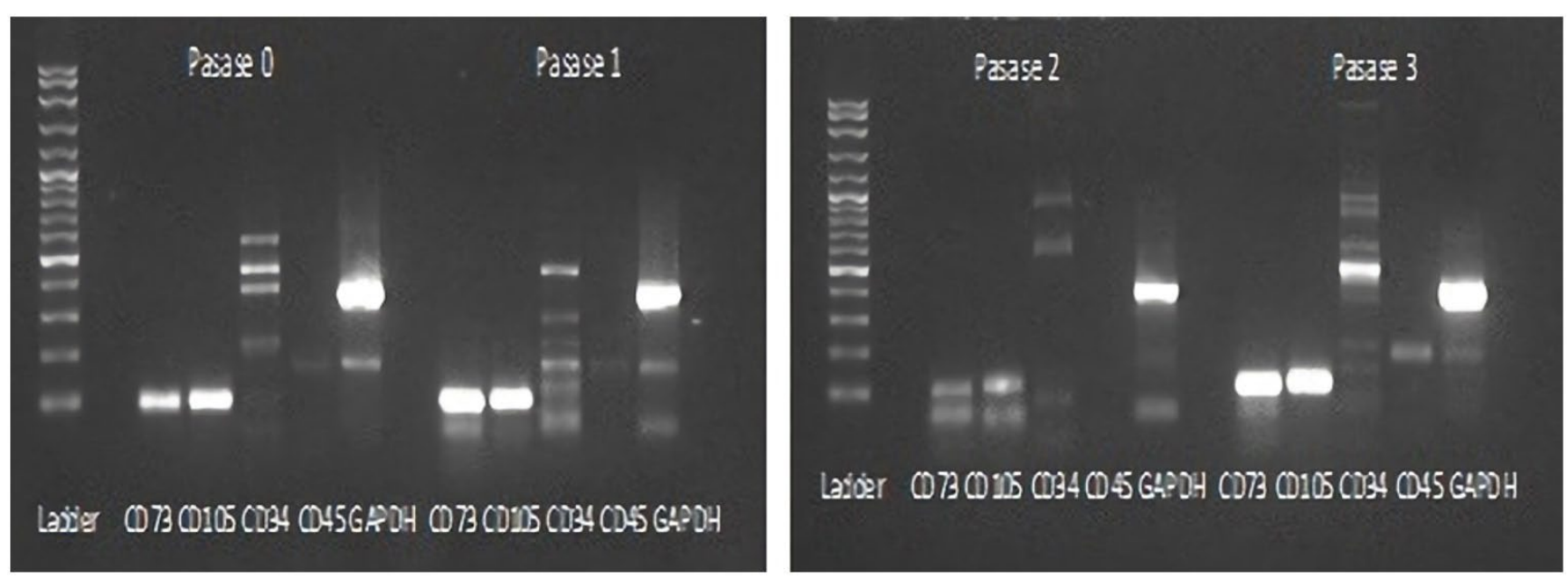

Figure 2. PCR amplicons for mesenchymal stem cell markers from M. nemestrina bone marrow. CD73 (101 bp), CD105 (104 bp), CD34 (380 bp), CD45 (167 bp), and GAPDH (352 bp) in passages 0-3
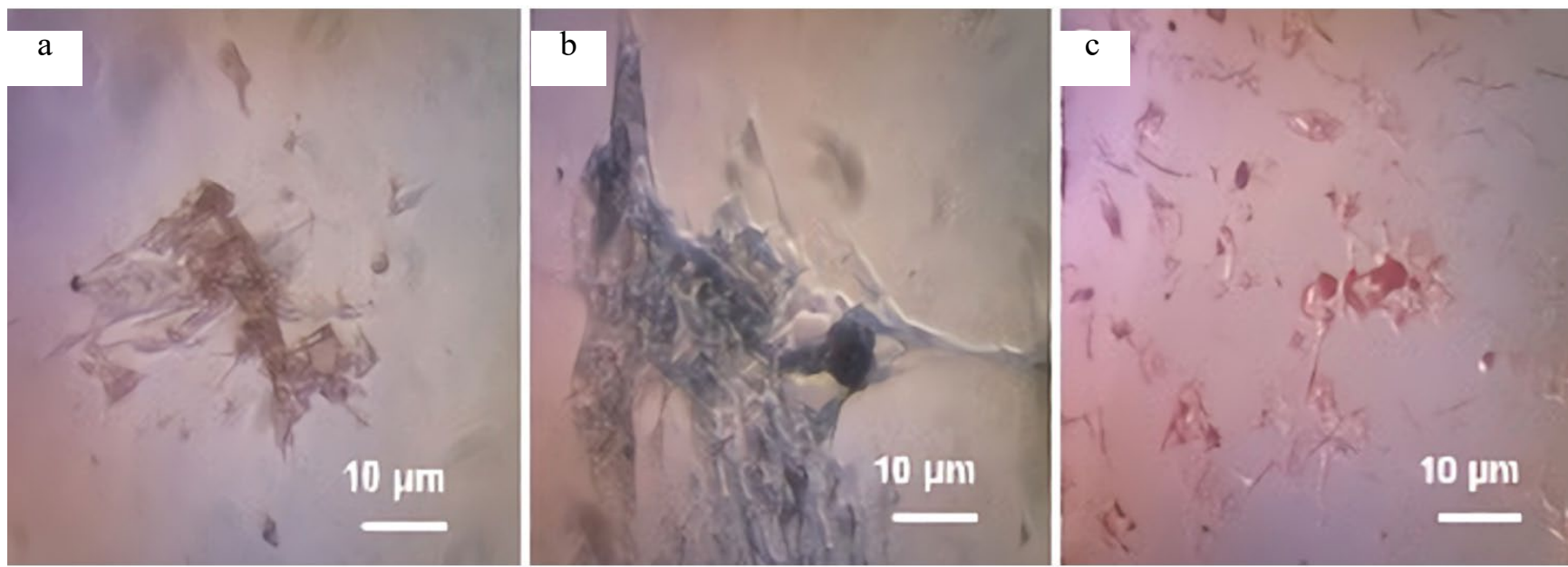

Figure 3. (a) Osteogenic differentiation shown with alizarin red staining, (b) chondrogenic differentiation shown with Alcian blue staining, (c) adipogenic differentiation shown with oil red 0 staining. Bar scale of $10 \mu \mathrm{m}$

chondrocytes were stained with Alcian blue to visualize chondrocyte nodules. Lastly, adipogenic differentiation produced cells of cuboidal morphology containing lipid-droplet deposits that were detectable using oil red $\mathrm{O}$ staining.

\subsection{Cardiomyocytic Differentiation Cardiac Electrical Cells Connexin}

Differentiation of cardiomyocytes, induced by the administration of 5-aza demethylation agents, produced morphological changes that resulted in elongated cells that attached to each other with a certain gap (Figure 4). By day 21, almost all cells had elongated morphology and expressed $\mathrm{Cx} 40$ and Cx43 proteins as detected by immunocytochemical staining of the culture.

\section{Discussion}

The volume of bone marrow aspirates obtained from $M$. nemestrina did not correspond to the number of mononuclear cells isolated. Mn\#3 contained the 


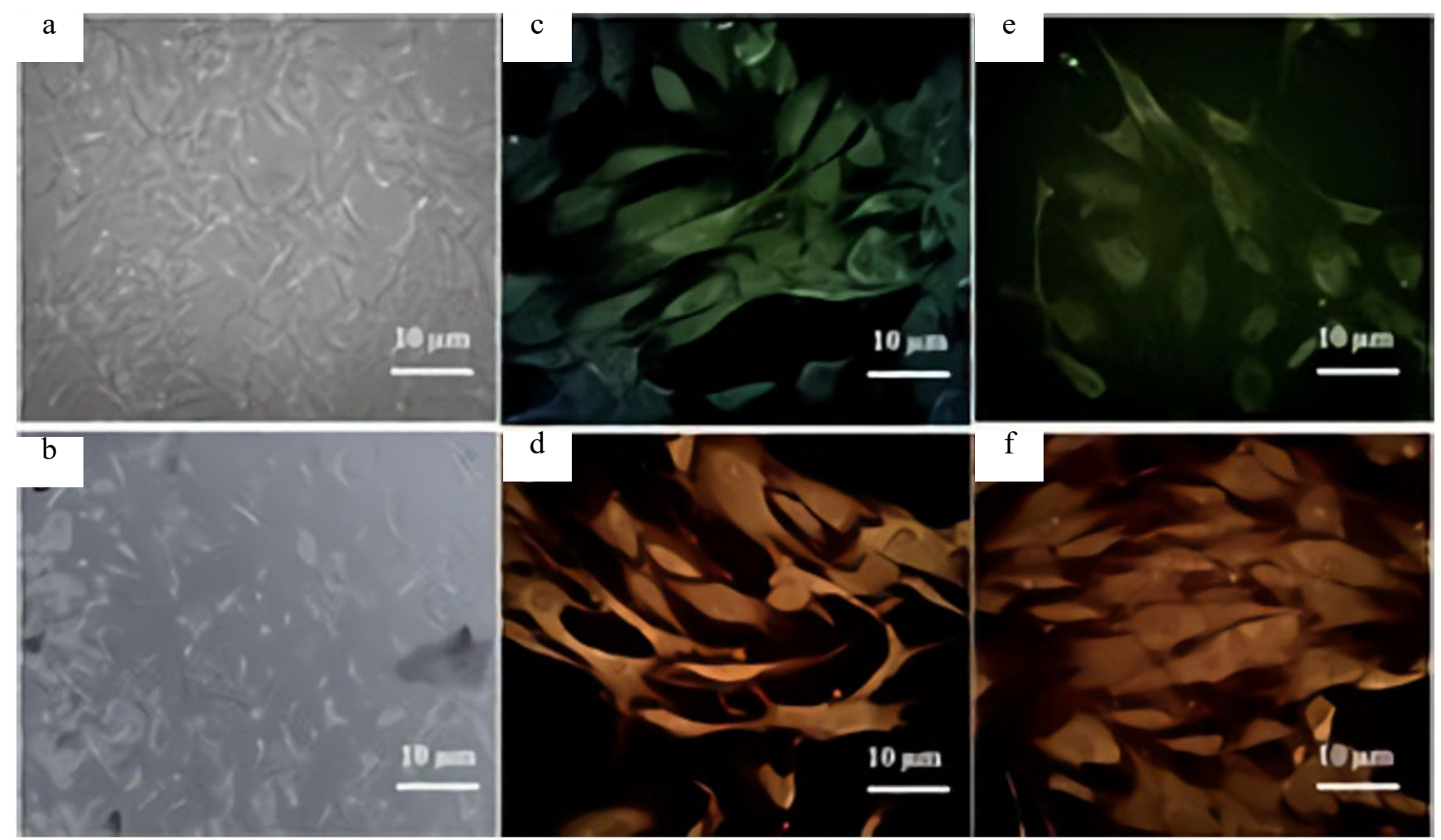

Figure 4. (a) Macaca nemestrina bone marrow mesenchymal stem cells, (b) cardiomyocyte differentiation, (c) positive Cx40, (d) negative Cx40, (e) positive Cx43, and (f) negative Cx43. Cx = connexin. Bar scale of $10 \mu \mathrm{m}$.

lowest number of mononuclear cells, possibly because of impaired haematopoiesis or the increased fat content of bone resulting in decreased haematopoiesis in this older animal, or that the animal had abnormalities in blood cells that resulted in anaemia and fewer immature lymphocytes, immature monocytes and progenitor cell populations (Henrich et al. 2015). Other possible causes might be slow fibroblast motility that eventually led to cellular death, an influence of Ficoll on fibroblast movement or abnormalities in cell cycle processes such as failure to repair DNA errors because of telomere instability (shortening of TTAGGG telomere ends), thus arresting the cell cycle and inducing apoptosis (Freshney 2010). Although mononuclear cell cultures from Mn\#1, Mn\#2, and Mn\#4 were $70-80 \%$ confluent by day 7 of culture, Mn\#3 cells did not grow and develop.

Confluence occurs when cells are able to grow because of their intercellular relationships, and the spindle morphology of fibroblast cells reflects the morphology of mesenchymal stem cells (Pittenger et al. 1999). Cell viability greater than $90 \%$ reflects healthy cells that are able to proliferate. The number of cells obtained from confluent cultures was $>4.8$ million, and it was hoped that the cells would be able to survive until the next passage.
Besides the spindle shape of fibroblasts, other characteristics of mesenchymal stem cells were observed; e.g. attachment to the growth surface, self-renewability and ability to differentiate into several cell types (Dominici et al. 2006). During the subculture process, cell growth was found to be slow during passages 1 and 2 , then slightly faster in passage 3. This is considered natural because normal tissue cells that are not of tumour origin will adapt to their new environment.

The PDT of cells increased from the first to the third passage, and division ultimately stopped after the third passage. PDT was measured based on the time for doubling the number of cells in primary cell culture isolates in vitro, and growth was evaluated and expressed using cell lineage growth curves. These curves consist of four phases, starting with the reseeding phase and the lag phase that lasts up to 48 hours. During this phase, the cells recover from trypsinization to build the cytoskeleton and rebuild the extracellular matrix (Teti 1992). The cytoskeleton connects thick layers of actin, intermediate filament tissue and microtubules throughout the cell, and regulates intracellular signal transduction. The extracellular matrix regulates cell functions such as adhesion, migration, proliferation, 
and differentiation. The lag process facilitates and propagates the intercellular connection, resulting in cells entering a new cycle. The cells then enter the log (exponential) phase or the population multiplication phase depending on the characteristics of each cell. In this phase, the effects of drugs, chemicals, immunomodulators and free radicals can stimulate or inhibit cell growth. The last phase is the stationary phase, in which the cell population is very dense and the substrate collects almost all metabolites, which results in a decrease in cell growth rates to almost zero (Assanga and Lujan 2013).

To confirm that the stem cells from bone marrow aspirates of $M$. nemestrina were of mesenchymal and not haematopoietic lineage, PCR amplification of mRNA isolated from the cells was performed, with products visualized by electrophoresis. Messenger RNA is part of the large family of RNA molecules that convey the genetic information of DNA to the ribosome by encoding a sequence of amino acids by means of three base-pair codons. The mRNA extraction process is effective if the buffer solution contains no $\mathrm{Na}^{+}, \mathrm{K}^{+}$or Tris- $\mathrm{HCl}$ at neutral $\mathrm{pH}$, resulting in the dissociation of the protein poly (A) and the release of RNA molecules from the protein (Brawerman 1974). We investigated the expression of CD73 and CD105, markers of mesenchymal stem cells that play roles in the cytoskeleton and cell morphology. CD73 is a long-term immunomodulator that allows recovery of damaged tissue through hydrolysis of adenosine triphosphate to adenosine monophosphate (MonguióTortajada et al. 2017) while CD105 expression is used to differentiate mesenchymal stem cells from other cell populations and does not occur in chondrogenic progenitors.

The electrophoretic bands for CD34 and CD45 doubled in length in passage 2 . This may be due to several factors, e.g. genetic inheritance, cis/trans replication environment (Bryois et al. 2017), variation of $6-8$ bp in the length of transcribed genes (Yilmaz and Grotewold 2010) or impaired post-transcriptional excision of introns and ligation of exons to yield the final mRNA(Sibley 2014). Unlike the cells of haematopoietic lineage, the population of mesenchymal stem cells are identified as not expressing CD34 and CD45, which are markers of haematopoietic progenitor cells and leukocytes, respectively.

Mesenchymal stem cells can further differentiate into osteogenic, chondrogenic and adipogenic stem cell lineages. Their differentiation into the corresponding lineage using appropriate culture media was successfully achieved in this study. Osteogenic differentiation results in cells with long cuboid ends that are larger than mesenchymal stem cells, and upon further differentiation into osteocytes, calcium-rich deposits are seen with alizarin red staining (Shah et al. 2016).

Chondrogenic differentiation is influenced by intrinsic and extrinsic factors such as beta growth factors with three type I receptors and three type II receptors. These influences, via cell proliferation, extracellular matrix production and cellular morphology yielded chondrogenic cells that were shortened, enlarged and rounded at one end. Alcian blue staining of these cells demonstrated chondrocyte nodules because cartilage is soluble in alkaline and glycerol (Terry et al. 2000).

Adipogenic differentiation was confirmed by oil red O staining for lipid-droplet deposits. Components of the different culture media, namely genetic, epigenetic and growth factors, directed morphogenesis along each differentiation pathway. The process of stem cell differentiation is thought to be caused by both internal and external factors (Birmingham et al. 2012).

In this study, we also tried to differentiate bone marrow mesenchymal stem cells of M. nemestrina into the cardiomyocytic lineage using 5-aza demethylation agents. The 5-aza compound is a cytosine analogue, and altered cell morphology by the seventh day, although the cultures still contained cells of mesenchymal stem cell morphology and some dead cells (Antonitsis et al. 2007) By day 21, almost all cells had an elongated morphology and expressed $\mathrm{Cx} 40$ and $\mathrm{Cx} 43$ proteins as detected by immunocytochemical staining of the culture. The percentage of cells expressing $\mathrm{Cx}$ (a gap junction isoform) in the myocardium is around $75 \%$, and where it forms an intercellular junction that connects the cardiomyocytes. Gap junctions, adherent junctions and desmosomes connect the intercalated disc lining covering the cardiomyocytes. The role of gap junctions in triggering myofibril contraction is mediated by the presence of charge in the $\mathrm{Cx}$ protein subunits that make up the gap junction itself (Valiunis et al. 2001).

There is still debate about the roles of $\mathrm{Cx} 40$ and Cx43 in the heart's electrical system. In one study, Cx43 was not expressed and only a small amount of Cx40 was expressed in the centre of sinoatrial and atrioventricular nodes (Boyett et al. 2006). In another study, Cx40 was mostly found in the atrioventricular node, His bundles and a small portion in the atrial and ventricular cardiomyocytes, whereas Cx43 
was mostly found in the atrial cardiomyocytes, ventricular cardiomyocytes and a small portion in the atrioventricular node and His bundles (Temple et al. 2013). Both studies employed immunohistochemical techniques rather than the immunocytochemistry techniques used in this study.

Bone marrow mesenchymal stem cells of $M$. nemestrina can be isolated and grown in vitro, with substrate attachment and assume spindle morphology similar to that of fibroblasts. These bone marrow mesenchymal stem cells could be subcultured up to passage 3. They expressed CD73 and CD105, which are bone marrow mesenchymal stem cell markers, and did not express the haematopoietic stem cell markers CD34 and CD45. We demonstrated that bone marrow mesenchymal stem cells could be differentiated into osteocytes, chondrocytes, adipocytes, and cardiomyocytes. The expression of $\mathrm{Cx} 40$ and $\mathrm{Cx} 43$, crucial components of the cardiac conduction system, was identified by immunocytochemical staining. Culture techniques for bone marrow stem cells of $M$. nemestrina need to be developed further to obtain a greater number of cells and higher passaging ability.

\section{Conclusion}

Bone marrow mesenchymal stem cells of $M$. nemestrina can be isolated and grown in vitro, could be differentiated into osteocytes, chondrocytes, adipocytes and cardiomyocytes, with expressed CD73 and CD105, and did not expressed CD34 and CD45. So the expression of $\mathrm{Cx} 40$ and $\mathrm{Cx} 43$, crucial components of the cardiac conduction system, was identified by immunocytochemical staining too.

\section{Acknowledgements}

We thank Silmi Mariya and Permanawati for their contributions and the Bogor Primates Research Center of Bogor Agricultural University for providing equipments, facilities, permissions, and ethical clearance for this experiments.

\section{References}

Antonitsis Pet al. 2007. In vitro cardiomyogenic differentiation of adult human bone marrow mesenchymal stem cells. The role of 5-azacytidine. Interact Cardiovasc Thorac Surg 6:593-597.
Assanga I, Lujan L. 2013. Cell growth curves for different cell lines and their relationship with biological activities. Int J Biotechnol Mol Biol Res 4:60-70.

Birmingham E et al. 2012. Osteogenic differentiation of mesenchymal stem cells is regulated by osteocyte and osteoblast cells in a simplified bone niche. Eur Cells Mater 23:13-27.

Boyett MR et al. 2006. Connexins in the sinoatrial and atrioventricular nodes. Adv Cardiol 42:175-197.

Brawerman G. 1974. The isolation of RNA from mammalian cells. Methods Cell Biol 7:1-22.

Bryois J et al. 2017. Time-dependent genetic effects on gene expression implicate aging processes. Genome Res 27:545-552.

Carlsson HE et al. 2004. Use of primates in research: a global overview. Am J Primatol 63:225-237.

Dominici M et al. 2006. Position paper. Minimal criteria for defining multipotent mesenchymal stromal cells. The international society for cellular therapy position statement. Cytotherapy 8:315-317.

Friedenstein AJ. 1989. Stromal-hematopoietic interrelationships: Maximov's ideas and modern models. Haematol Blood Transfus 32:159-167.

Freshney RI. 2010. Culture of Animal Cells. A Manual of Basic Technique and Specialized Applications, 6th ed. New Jersey: Wiley-Blackwell.

Henrich D et al. 2015. Characterization of bone marrow mononuclear on biomaterials for bone tissue engineering. Biomed Res Int 2015:762407.

Monguió-Tortajada M et al. 2017. Mesenchymal stem cells induce expression of CD73 in human monocytes in vitro and in a swine model of myocardial infarction in vivo. Front Immunol 8:1-13.

Pittenger MF et al. 1999. Multilineage potential of adult human mesenchymal stem cells. Science 284:143-147.

Shah KM et al. 2016. Laboratory methods. Osteocyte isolation and culture methods. BoneKEy Reports 5:1-9.

Sibley CR. 2014. Regulation of gene expression through production of unstable mRNA isoforms. Biochem Soc Trans 42:1196-1205.

Temple IP et al. 2013. Connexins and the atrioventricular node. Heart Rhythm 10:297-304.

Terry DE et al. 2000. Differential use of Alcian blue and toluidine blue dyes for the quantification and isolation of anionic glycoconjugates from cell cultures: application to proteoglycans and a high-molecular-weight glycoprotein synthesized by articular chondrocytes. Anal Biochem 285:211-219.

Teti A. 1992. Regulation of cellular functions by extracellular matrix. J Am Soc Nephrol 10:83-87.

Valiunas V et al. 2001. Gap junction channels formed by coexpressed connexin 40 and connexin 43. Am JPhysiol Heart Circ Physiol 281:1675-1689.

van Kempen MJ et al. 1995. Differential connexin distribution accommodates cardiac function in different species. Microsc Res Tech 31:420-436.

Yilmaz A, Grotewold E. 2010. Components and Mechanisms of Regulation of Gene Expression. In: Ladunga I (Eds.). Computational Biology of Transcription Factor Binding. Lincoln Nebraska: Humana Press. pp. 23-32.

Zhang X et al. 2017. Differentiation and characterization of rhesus monkey atrial and ventricular cardiomyocytes from induced pluripotent stem cells. Stem Cell Res 20:21-29. 